T. H. Steele, Department of Mathematics, Weber State University, Ogden, UT 84408-1702, e-mail: thsteele@@weber.edu

\title{
TOWARDS A CHARACTERIZATION OF $\omega$-LIMIT SETS FOR LIPSCHITZ FUNCTIONS
}

\begin{abstract}
Recent research has shown that there is a significant cleavage between the structure of $\omega$-limit sets for continuous functions, and the structure of $\omega$-limit sets for Lipschitz functions. While every non-empty nowhere dense compact set is an $\omega$-limit set for a continuous function, most of these sets cannot be an attractor for a Lipschitz function. When one considers the topological structure of these two classes of $\omega$-limit sets, however, there is no such divergence. In this paper we investigate the necessarily measure based structural differences between these two classes of sets. We then use these results to work towards a characterization of $\omega$-limit sets for Lipschitz functions.
\end{abstract}

\section{Introduction}

A set $E$ is called an $\omega$-limit set, or an attractor, for a continuous function $f$ mapping a compact interval $I$ into itself if there exists an $x$ in $I$ such that $E=\omega_{f}(x)$ is the cluster set of the sequence $\left\{f^{n}(x)\right\}_{n=0}^{\infty}$. Recent work by Bruckner, Ceder and Smítal in [BC], [BS1] and [BS2] not only completely characterizes $\omega$-limit sets for continuous functions, but also demonstrates how the structure of these attractors is affected by imposing conditions on the chaotic behavior of the function in question. In [BSt] we show that there is a significant cleavage between the structure of attractors for continuous functions, and the structure of attractors for Lipschitz functions. We furnish

Key Words: omega limit set, Lipschitz function

Mathematical Reviews subject classification: Primary: 26A18

Received by the editors September 29, 1995 
the class $\mathcal{K}$ of non-empty closed subsets of $I=[0,1]$ with the Hausdorff metric $d$, and from this complete metric space develop a dense $\mathrm{G}_{\delta}$ subset comprised of Cantor sets that cannot be attractors for any Lipschitz function. This is in marked contrast to the continuous case, since any Cantor set is an $\omega$-limit set for some continuous function with zero topological entropy [BS2].

Our goal in this paper is to gain a better understanding of the structure of attractors for Lipschitz functions. To this end, we develop a characterization of homoclinic attractors for Lipschitz functions, as well as a sufficient condition for a nowhere dense perfect set to be an attractor for a Lipschitz function with zero topological entropy.

After a short preliminary section in which we develop a few basic definitions, establish notation, and record some simple results, we lay the foundation of our main results in section three. There we study necessary and sufficient conditions for a nowhere dense compact set to be mapped onto another by a Lipschitz function. We then apply these findings to the structure of $\omega$-limit sets in section four, in which we develop our main results.

\section{Preliminaries}

In developing their characterization of $\omega$-limit sets for continuous functions, Bruckner and Smítal make extensive use of the notion of a homoclinic trajectory. Let $M$ be a nowhere dense compact set, with $A=\left\{a_{0}, a_{1}, \ldots, a_{k-1}\right\} \neq \emptyset$ a set of limit points of $M$. For $i=0,1, \ldots, k-1$, we take $\left\{M_{n}^{i}\right\}_{n=0}^{\infty}$ to be a system of non-empty pairwise disjoint compact subsets of $M$ such that $M \backslash \bigcup_{i, n} M_{n}^{i}=A$ and $\lim _{n \rightarrow \infty} M_{n}^{i}=a_{i}$ for any $i$. Moreover, let $f: M \rightarrow M$ be a continuous map with $A$ a $k$-cycle of $f$ such that $f\left(a_{i}\right)=a_{i-1}$ for $i>0$ and $f\left(a_{0}\right)=a_{k-1}$. If $f\left(M_{n}^{i}\right)=M_{n}^{i-1}$ for $i>0$ and any $n, f\left(M_{n}^{0}\right)=M_{n-1}^{k-1}$ for $n>0$, and $f\left(M_{0}^{0}\right)=a_{k-1}$, then $M$ is called a homoclinic set (of order $k$ ) with respect to $f$.

Since we will also be discussing attractors of Lipschitz functions with zero topological entropy, we will have need to make use of the following theorem due to Smítal [S].

Theorem 2.1 (Smítal's Theorem) Let $f: I \rightarrow I$ be a continuous function with zero topological entropy, and let $E$ be an infinite attractor of $f$. Then there is a sequence $\left\{J_{k}\right\}_{k=1}^{\infty}$ of $f$-periodic intervals so that, for any $k$,

(1) $J_{k}$ has period $2^{k}$;

(2) $J_{k+1} \cup f^{2^{k}}\left(J_{k+1}\right) \subseteq J_{k}$; 
(3) $E \subseteq \bigcup_{i=1}^{2^{k}} f^{i}\left(J_{k}\right)$;

(4) $E \cap f^{i}\left(J_{k}\right) \neq \emptyset$ for every $i$.

Both Smítal's Theorem and the notion of a homoclinic trajectory have portions of the attractor mapping over other portions. Central to our investigation, then, is a development of necessary and sufficient conditions which insure that, for two nowhere dense compact sets $E$ and $F$, there exists a Lipschitz function $f: E \rightarrow F$ so that $f(E)=F$. An important tool in this investigation is the generalized Hausdorff measure. Let $\Phi$ denote the set of functions $\phi$ that are continuous and increasing on $I=[0,1]$ with $\phi(0)=0$. For $\phi \in \Phi$, set

$$
\begin{array}{r}
\mu_{\phi, n}(E)=\inf \left\{\Sigma \phi\left(\left|I_{i}\right|\right): E \subseteq \bigcup I_{i}, \text { and } I_{i}\right. \text { is an open interval } \\
\text { of length } \left.\left|I_{i}\right| \leq \frac{1}{n}\right\}
\end{array}
$$

Then $\mu_{\phi}=\lim _{n \rightarrow \infty} \mu_{\phi, n}$ defines a measure on the Borel sets in $I$. In what follows, we are concerned primarily with closed sets.

We will call a portion $P$ of a closed set $E$ a nonempty set of the form $P=E \cap J$, where $J$ is an open interval. If $J$ has rational endpoints, we will call $P$ a rational portion of $E$. We denote the class of Lipschitz functions on $I$ by Lip, and those $f \in$ Lip with Lipschitz constant $M$ by Lip $M$. We use $\lambda E$ to denote the Lebesgue measure of $E$, and let $|E|$ be the diameter of $E$. Finally, we let $E \rightarrow F$ indicate the existence of $f \in \operatorname{Lip}$ such that $f(E)=F$; if we have $f \in \operatorname{Lip} M$, we write $E \stackrel{M}{\rightarrow} F$.

\section{Intermediate Results}

The goal of this section is to develop necessary and sufficient conditions on nowhere dense compact sets $E$ and $F$ which insure the existence of a Lipschitz function $f: E \rightarrow F$ so that $f(E)=F$.

We begin with the following elementary observation from [BSt]:

Proposition 3.1 If $\phi \in \Phi$ and $f \in \operatorname{Lip} M$ for some $M \in \mathbf{N}$, then $\mu_{\phi} f(E) \leq$ $M \mu_{\phi}(E)$ for every Borel set $E$.

From Proposition 3.1, we see that the $\mu_{\phi}$ measures of $E$ and $F$ must be closely related if $E \rightarrow F$. In our next proposition we develop a much finer bound on this growth in measure provided that the gauge function $\phi$ is concave. 
Proposition 3.2 Let $\phi \in \Phi$ such that $\phi$ is concave, with $f \in \operatorname{Lip} M$. Then $S=\lim _{\delta \rightarrow 0} \frac{\phi(M \delta)}{\phi(\delta)}$ exists, and $\mu_{\phi} f(E) \leq S \mu_{\phi}(E)$.

Proof. It suffices to show that $\mu_{\phi}(M E)=S \mu_{\phi}(E)$. Since $\phi$ is concave, $\lim _{\delta \rightarrow 0} \frac{\phi(M \delta)}{\phi(\delta)}$ exists and is bounded, so that for any $\epsilon>0$ there exists $n(\epsilon) \epsilon$ $\mathbb{N}$ such that $n \geq n(\epsilon)$ implies $\frac{\phi(M / n)}{\phi(1 / n)} \leq S+\epsilon$. Recall that $\mu_{\phi, n}(E)=$ $\inf \left\{\sum_{i} \phi\left(\left|I_{i}\right|\right): E \subseteq \bigcup_{i} I_{i}\right.$, and $\left.\left|I_{i}\right| \leq \frac{1}{n}\right\}$. Fixing $\epsilon>0$ thus yields $\mu_{\phi, n}(M E) \leq$ $\sum_{i} \phi\left(\left|M I_{i}\right|\right) \leq(S+\epsilon) \sum_{i} \phi\left(\left|I_{i}\right|\right)$ for any $\frac{1}{n}$ cover of $E$ for which $n \geq n(\epsilon)$. Letting $n$ go to infinity gives us

$$
\mu_{\phi}(M E) \leq S \mu_{\phi}(E) .
$$

Suppose $\left\{I_{i}\right\}$ is a cover of $M E$ such that $\left|I_{i}\right| \leq \frac{M}{n}$ for all $i$, so that $\left\{\frac{1}{M} I_{i}\right\}$ is a $\frac{1}{n}$ cover of $E$. Since $S=\lim _{\delta \rightarrow 0} \frac{\phi(M \delta)}{\phi(\delta)}$, for any $\epsilon>0$ there exists $N(\epsilon) \in \mathbb{N}$ so that $n \geq N(\epsilon)$ implies $\frac{\phi(M / n)}{\phi(1 / n)} \geq S-\epsilon$. Fix $\epsilon>0$. Then $\mu_{\phi, \frac{1}{n}}(E) \leq \sum_{i} \phi\left(\left|\frac{I_{i}}{M}\right|\right) \leq$ $\frac{1}{S-\epsilon} \sum_{i} \phi\left(I_{i}\right)$ for any $\frac{M}{n}$ cover of $M E$ such that $n \geq N(\epsilon)$. Letting $n$ go to infinity gives us $\mu_{\phi}(E) \leq \frac{1}{S} \mu_{\phi}(M E)$, so that

$$
S \mu_{\phi}(E) \leq \mu_{\phi}(M E) .
$$

Putting (1) and (2) together, we have $S \mu_{\phi}(E)=\mu_{\phi}(M E)$.

Our next two results show that given any $\phi \in \Phi$, there exists a $\tilde{\phi} \in \Phi$ which is concave, and has the property that for any $E \in \mathcal{K}, \mu_{\phi}(E)=\mu_{\tilde{\phi}}(E)$. Thus, when dealing with $\omega$-limit sets, we will be able to presume that our gauge functions are concave, and make good use of our previous proposition.

Lemma 3.3 Let $\phi \in \Phi$. If the lower right Dini derivative of $\phi$ is finite, for instance $D_{+} \phi(0)=s<\infty$, and $E \in \mathcal{K}$ for which $\lambda E=c$, then $\mu_{\phi}(E)=s c$.

Proof. Let $\left\{x_{n}\right\} \subseteq[0,1]$ such that $\lim _{n \rightarrow \infty} x_{n}=0$, and $\lim _{n \rightarrow \infty} \frac{\phi\left(x_{n}\right)-\phi(0)}{x_{n}-0}=$ $\lim _{n \rightarrow \infty} \frac{\phi\left(x_{n}\right)}{x_{n}}=s$. Then, for any $\epsilon>0$, there exists $N \in \mathbb{N}$ so that $m>N$ implies $\frac{\phi\left(x_{m}\right)}{x_{m}}<s+\epsilon$. Since $\lambda E=c$, for any $\delta>0$ there exists an open cover 
$\left\{I_{n}\right\}_{n=1}^{M}$ of $E$ such that $\sum_{n=1}^{M} \lambda I_{n}<c+\delta$. Now, let us fix $k \in \mathbb{N}$. Then there exists $N_{k} \in \mathbb{N}$ such that $m>N_{k}$ implies $x_{m} \leq 1 / k$ and $\frac{\phi\left(x_{n}\right)}{x_{n}}<s+\epsilon$. Now, let $\left\{I_{n}\right\}_{n=1}^{M}$ be a sequence of open intervals that cover $E$, with $\sum_{n=1}^{M} \lambda I_{n}<c+\delta$, and $\left|I_{n}\right|=x_{j_{n}}$ for some $j_{n}>N_{k}$ and for all $n=1,2, \ldots, M$. Then $\mu_{\phi, k}(E) \leq$ $\sum_{n=1}^{M} \phi\left(\left|I_{n}\right|\right)=\sum_{n=1}^{M} \phi\left(x_{j_{n}}\right)<(s+\epsilon) \sum_{n=1}^{M} x_{j_{n}}$, and since $\sum_{n=1}^{M} x_{j_{n}}=\sum_{n=1}^{M} \lambda I_{n}<c+\delta$, we have $\mu_{\phi, k}(E)<(s+\epsilon)(c+\delta)=s c+s \delta+\epsilon c+\epsilon \delta$. Thus, $\mu_{\phi}(E) \leq s c$. But since $\lambda E=c$ and $D_{+} \phi(0)=s$, we've also that $\mu_{\phi}(E) \geq s c$, so that, in fact, we have $\mu_{\phi}(E)=s c$.

Lemma 3.4 Suppose $\phi \in \Phi$ with $D_{+} \phi(0)=\infty$. Then there exists $\tilde{\phi} \in \Phi, \tilde{\phi}$ concave, such that $\mu_{\phi}(E)=\mu_{\tilde{\phi}}(E)$ for all $E$ in $\mathcal{K}$.

Proof. We first construct our function $\tilde{\phi}$. For any $n$, let $A_{n}=\{x \in[0,1]$ : $\left.\frac{\phi(x)}{x}=n\right\}$, and let $x_{n}=\min A_{n}$. Now, let $B_{n}=\left\{y \in\left[x_{n+1}, x_{n}\right]:\right.$ there exists $\epsilon>0$ such that $x \in B_{\epsilon}(y)$ implies $\left.\frac{\phi(x)}{x} \geq \frac{\phi(y)}{y}\right\}$. If $B_{n} \neq \emptyset$, let $B_{n}{ }^{\prime} \subseteq B_{n}$ with $y \in B_{n}{ }^{\prime}$ if $\frac{\phi(y)}{y} \leq \frac{\phi(x)}{x}$ for every $x \in B_{n}$, and let $x_{n}{ }^{\prime}=\min B_{n}{ }^{\prime}$. If $B_{n}=\emptyset$, let $x_{n}{ }^{\prime}=x_{n}$. Let $y_{1}=x_{1}{ }^{\prime}, y_{2}=x_{2}{ }^{\prime}$ and let $y_{3}$ be the first element of $\left\{x_{n}{ }^{\prime}\right\}_{n=3}^{\infty}$ such that the slope between $\left(y_{2}, \phi\left(y_{2}\right)\right)$ and $\left(x_{n}{ }^{\prime}, \phi\left(x_{n}{ }^{\prime}\right)\right)$ is greater than the slope between $\left(y_{1}, \phi\left(y_{1}\right)\right)$ and $\left(y_{2}, \phi\left(y_{2}\right)\right)$. In general, if we have defined $y_{1}, y_{2}, \ldots y_{n}=x_{m}{ }^{\prime}$, let $y_{n+1}$ be the first element of $\left\{x_{i}{ }^{\prime}\right\}_{i=m+1}^{\infty}$ such that the slope between $\left(y_{n}, \phi\left(y_{n}\right)\right)$ and $\left(x_{i}{ }^{\prime}, \phi\left(x_{i}{ }^{\prime}\right)\right)$ is greater than the slope between $\left(y_{n-1}, \phi\left(y_{n-1}\right)\right)$ and $\left(y_{n}, \phi\left(y_{n}\right)\right)$. Let $\tilde{\phi}(x)=\phi(x)$ if $x \in\left\{y_{n}\right\}$ and extend $\tilde{\phi}$ linearly on the intervals $\left(y_{n+1}, y_{n}\right)$. Finally, let $\tilde{\phi}(x)=\tilde{\phi}\left(y_{1}\right)$ for $x \in\left[y_{1}, 1\right]$.

Now, since $\phi(x) \geq \tilde{\phi}(x)$ on $[0,1]$, it follows that $\mu_{\phi}(E) \geq \mu_{\tilde{\phi}}(E)$ for all $E$ in $\mathcal{K}$. Similarly, since $\left\{y_{n}\right\} \varsubsetneqq[0,1]$, we've that $\mu_{\phi \mid\left\{y_{n}\right\}}(E) \geq \mu_{\phi}(E)$ for all $E$ in $\mathcal{K}$. Thus, we need only establish that $\mu_{\phi \mid\left\{y_{n}\right\}}(E)=\mu_{\tilde{\phi}}(E)$ for all $E$ in $\mathcal{K}$ for our desired conclusion to follow.

To this end, let $\left\{z_{n}\right\}$ be any countable collection of points in $[0,1]$ that have the origin as an accumulation point. It suffices to show that for any $E$ in $\mathcal{K}$ we have

$$
\mu_{\tilde{\phi} \mid\left\{z_{n}\right\}}(E)=\mu_{\phi \mid\left\{y_{n}\right\}}(E) .
$$

Since we may assume that $\left\{y_{n}\right\} \subseteq\left\{z_{n}\right\}$, as the union of any two countable sets is itself countable, we have that

$$
\mu_{\tilde{\phi} \mid\left\{z_{n}\right\}}(E) \leq \mu_{\phi \mid\left\{y_{n}\right\}}(E) \text { for any } E \text { in } \mathcal{K} .
$$


To get the opposite inequality, fix $k \in \mathbb{N}$ and $\epsilon>0$, and take $\left\{I_{n}\right\}_{n=1}^{N}$ to be an open cover for a particular set $E$ so that $\sum_{n=1}^{N} \phi\left(\left|I_{n}\right|\right)=\sum_{n=1}^{N} \tilde{\phi}\left(\left|I_{n}\right|\right) \geq$ $\mu_{\phi \mid\left\{y_{n}\right\}, k}(E)>\sum_{n=1}^{N} \phi\left(\left|I_{n}\right|\right)-\epsilon$. Let $p(k) \in \mathbb{N}$ such that $\frac{1}{p(k)}<\min \left\{I_{n}\right.$ : $n=1,2, \ldots, N\}$. Then, since $\tilde{\phi}$ is concave, we have $\mu_{\tilde{\phi} \mid\left\{z_{n}\right\}, p(k)}(E)+\epsilon \geq$ $\mu_{\phi \mid\left\{y_{n}\right\}, k}(E)$. As $k$ goes to infinity, we have $\mu_{\tilde{\phi} \mid\left\{z_{n}\right\}}(E)+\epsilon \geq \mu_{\phi \mid\left\{y_{n}\right\}}(E)$. Since our $\epsilon$ was arbitrary, we have $\mu_{\tilde{\phi} \mid\left\{z_{n}\right\}}(E) \geq \mu_{\phi \mid\left\{y_{n}\right\}}(E)$.

Suppose we have two nowhere dense compact sets $E$ and $F$ so that $E \stackrel{M}{\rightarrow} F$. From Proposition 3.2, we know that $\mu_{\phi}(F) \leq S_{\phi}^{M} \mu_{\phi}(E)$ for all $\phi \in \Phi$, where $S_{\phi}^{M}=\lim _{\delta \rightarrow 0} \frac{\phi(M \delta)}{\phi(\delta)}$. A natural question for us to now ask is the following. If we have two sets that are non-trivially compatible for some $\alpha \in \Phi$, must they then be compatible for all $\phi \in \Phi$ ? This is the subject of our next result.

Proposition 3.5 Let $E$ and $F$ be elements of $\mathcal{K}$. If there exists $\phi \in \Phi$ such that $\mu_{\phi}(E)$ and $\mu_{\phi}(F)$ are both non-zero and finite, then for all $\alpha \in \Phi, \mu_{\alpha}(E)$ and $\mu_{\alpha}(F)$ are both either zero, non-zero and finite, or infinite.

Proof. Let $A$ and $B$ be positive real numbers so that $A<\mu_{\phi}(E)<B$ and $A<\mu_{\phi}(F)<B$. We prove our assertion by considering two cases.

CASE 1: Suppose there exists $\alpha \in \Phi$ such that $\mu_{\alpha}(E)=0$, but $\mu_{\alpha}(F)>0$. Since $\mu_{\alpha}(E)=0$ and $\mu_{\alpha}(E)=\lim _{n \rightarrow \infty} \mu_{\alpha, n}(E)$ where $\mu_{\alpha, n}(E)$ increases as $n$ increases, we have that $\mu_{\alpha, n}(E)=0$ for all $n$. Thus,

$$
\begin{aligned}
\mu_{\phi \circ \alpha, n}(E) & =\inf \left\{\sum_{i=1}^{M} \phi \circ \alpha\left(\left|I_{i}\right|\right): E \subseteq \bigcup_{i=1}^{M} I_{i},\left|I_{i}\right| \leq \frac{1}{n}\right\} \\
& \leq \inf \left\{M \phi\left(\sum \alpha\left(I_{i}\right)\right): E \subseteq \cup I_{i},\left|I_{i}\right|<\frac{1}{n}\right\} \\
& =0
\end{aligned}
$$

since $\phi \circ \alpha\left(\left|I_{i}\right|\right) \leq \phi\left(\sum_{i} \alpha\left(\left|I_{i}\right|\right)\right)$ for all $i$. Now, since $\mu_{\alpha}(F)>0$, we know that $\mathrm{D}_{+} \alpha(0)>0$. Let $f(x)=m x$, where $0<m<\mathrm{D}_{+} \alpha(0)$. Thus, $\mu_{\alpha} f(E) \leq$ $\mu_{\phi \circ \alpha}(E)=0$, but Proposition 2.4 of [BSt] implies $\mu_{\phi} f(E)>0$ since $\mu_{\phi}(E)>0$. Therefore, $\mu_{\alpha}(F)=0$, as well.

CASE 2: Suppose there exists $\sigma \in \Phi$ such that $\mu_{\sigma}(E)=\infty$, but $\mu_{\sigma}(F)<$ $\infty$. We assume that both $\phi$ and $\sigma$ are concave. Since $\mu_{\phi}(E)<B$, we know that for any $n, \mu_{\phi, n}(E)<B$ as well, as is the case with $F$. Since $\mu_{\sigma}(E)=\infty$, 
for any $M \in \mathbb{N}$ there exists an $N \in \mathbb{N}$ so that $n \geq N$ implies $\mu_{\sigma, n}(E)>M$. Let us fix $\epsilon>0$, set $r=\frac{\mu_{\sigma}(F)}{A}$, and let $N \in \mathbb{N}$ so that $n \geq N$ implies $\mu_{\sigma, n}(E)>$ $r B>r\left(\mu_{\sigma}(E)+\epsilon\right)$. Take $n_{1} \geq N$, and let $\left\{I_{i}\right\}$ be a finite sequence of open intervals such that $\bigcup_{i} I_{i} \supseteq E$ and $\left|I_{i}\right| \leq \frac{1}{n_{1}}$, for any $i$, with $\sum_{i} \phi\left(\left|I_{i}\right|\right)<B$. Then there exists $I_{1}{ }^{*} \in\left\{I_{i}\right\}$ such that $\frac{\sigma\left(\left|I_{1}{ }^{*}\right|\right)}{\phi\left(\left|I_{1}{ }^{*}\right|\right)}>r$. Let $n_{2}>\frac{1}{\left|I_{1}{ }^{*}\right|}$, and now take $\left\{I_{i}\right\}$ so that $\bigcup_{i} I_{i} \supseteq E$ and $\left|I_{i}\right| \leq \frac{1}{n_{2}}$ for any $i$, with $\sum_{i} \phi\left(\left|I_{i}\right|\right)<B$. Then there exists $I_{2}{ }^{*} \in\left\{I_{i}\right\}$ such that $\frac{\sigma\left(\left|I_{2}{ }^{*}\right|\right)}{\phi\left(\left|I_{2}{ }^{*}\right|\right)}>r$. We continue this process, developing the sequence $\left\{\left|I_{i}{ }^{*}\right|\right\}$ so that $\lim _{i \rightarrow \infty}\left|I_{i}{ }^{*}\right|=0$, and for any $i, \frac{\sigma\left(\left|I_{i}{ }^{*}\right|\right)}{\phi\left(\left|I_{i}{ }^{*}\right|\right)}>r$. From the proof of Lemma 3.4, we know that $\mu_{\phi}(E)=\mu_{\phi \mid\left\{z_{i}\right\}}(E)$ for any $E \in \mathcal{K}$, where $z_{i}=\left|I_{i}{ }^{*}\right|$ for each $i$. Similarly, we have $\mu_{\sigma}(E)=\mu_{\sigma \mid\left\{z_{i}\right\}}(E)$ as well. But $\frac{\sigma\left(z_{i}\right)}{\phi\left(z_{i}\right)}>r$ for any $i$ implies $\mu_{\phi \mid\left\{z_{i}\right\}}(F)>r \mu_{\phi}(F)>\mu_{\sigma}(F)$. Therefore, $\mu_{\sigma}(F)=\infty$ as well.

Now, let us suppose we have two compact nowhere dense sets $E$ and $F$ so that $f(E)=F$ for some $f$ in Lip $M$. Let $\left\{F_{n}\right\}$ be an enumeration of the clopen portions of $F$; that is, those portions which are both open and closed in the relative topology of $F$. To each $F_{n}$ there corresponds $f^{-1}\left(F_{n}\right)=E_{n}$, a clopen subset of $E$, such that

(1) $\frac{\mu_{\alpha}\left(F_{n}\right)}{\mu_{\alpha}\left(E_{n}\right)} \leq S_{\alpha}^{M}$ for all concave $\alpha \in \Phi$, and

(2) $\frac{\left|F_{n}\right|}{\left|E_{n}\right|} \leq M$

If, on the other hand, we suppose that $(p, q) \subset(\min F, \max F)$ is a complementary interval of $F$, then there exists $(r, s)$ a complementary interval of $E$ so that $(p, q) \subseteq f((r, s))$, and $|q-p| \leq M|s-r|(3)$.

Let us take $\left\{F_{n}^{p}\right\}$ and $\left\{F_{n}^{q}\right\}$ to be subsequences of $\left\{F_{n}\right\}$ such that $p \in F_{n}^{p}$ and $q \in F_{n}^{q}$ for each $n$ in $\mathbb{N}$, and $\left|F_{n}^{p}\right|$ and $\left|F_{n}^{q}\right|$ both go to zero as $n \rightarrow \infty$. Then if $f^{-1}\left(F_{n}^{p}\right)=E_{n}^{p}$, and we set $E^{p}=\cap_{n=1}^{\infty} E_{n}^{p}$, it follows that $f^{-1}(p)=E^{p}$. Thus, for any $x \in E^{p}$ and $y \in E^{q}$, we have that $M|x-y| \geq|p-q|$.

From our observation (3), we get the following interesting result.

Lemma 3.6 Let $\left\{b_{n}\right\}$ be an enumeration of the complementary intervals of $F$ such that $\left|b_{1}\right| \geq\left|b_{2}\right| \geq\left|b_{3}\right| \geq \cdots$, with $\left\{a_{n}\right\}$ an analogous enumeration 
for $E$. If $E \stackrel{M}{\rightarrow} F$, then there exists a subsequence $\left\{a_{m(n)}\right\} \subseteq\left\{a_{n}\right\}$ so that $\frac{\left|b_{n}\right|}{\left|a_{m(n)}\right|} \leq M$ for all $n \in \mathbb{N}$.

We are now in a position to develop the necessary and sufficient conditions on two nowhere dense compact sets $E$ and $F$ which insure $E \stackrel{M}{\rightarrow} F$. From our earlier discussion, we know that Lipschitz functions can only impose a certain amount of growth on both the measure and the length of any portion in the domain. The basic idea of our theorem, then, is to insure that the length and measure of portions of $E$ and $F$ are compatible with each other as well as with the Lipschitz constant $M$.

Theorem 3.7 Suppose $E$ and $F$ are both nowhere dense compact subsets of the unit interval, with $\left\{F_{n}\right\}$ an enumeration of the clopen portions of $F$. Then $E \stackrel{M}{\rightarrow} F$ if and only if to every $F_{k} \in\left\{F_{n}\right\}$ we can associate a clopen subset $E_{k} \subseteq E$ so that

(1) $\frac{\mu_{\alpha}\left(F_{k}\right)}{\mu_{\alpha}\left(E_{k}\right)} \leq S_{\alpha}^{M}$ for all concave $\alpha \in \Phi$,

(2) $\frac{\left|F_{k}\right|}{\left|E_{k}\right|} \leq M$,

(3) a. if $F_{k} \subseteq F_{j}$, then $E_{k} \subseteq E_{j}$

b. if $F_{k} \cap F_{j}=\emptyset$, then $E_{k} \cap E_{j}=\emptyset$

c. if $F_{n}=F_{k} \cup F_{j}$ is a disjoint union, then $E_{n}=E_{k} \cup E_{j}$ is a disjoint union,

(4) if $x \in E^{p}=\cap_{p \in F_{n}} E_{n}$ and $y \in E^{q}$, then $|x-y| \geq \frac{|p-q|}{M}$.

Proof. The necessity of our result is an immediate consequence of the existence of a Lip $M$ function $f$ such that $f(E)=F$. As for its sufficiency, we first note that condition (3) allows us to well define the set $E^{p}$ in (4). Thus we are able to define the function $f: E \rightarrow F$ where $x \in E^{t}$ implies $f(x)=t$. Condition (4) assures us that $f$ is $\operatorname{Lip} M$.

\section{Main Results}

With Theorem 3.7 we are now able to gain a good understanding of what it takes for a nowhere dense compact set to be a homoclinic attractor for a Lipschitz function. The critical aspect of these sets is that we are able to decompose them into compact subsets that not only develop a homoclinic 
trajectory but also can be mapped onto one another by a Lipschitz function with a given constant.

Theorem 4.1 Let $E \subseteq I$ be a nowhere dense compact set, with $f: E \rightarrow E$ a Lipschitz function of constant $N-\epsilon$ for some $\epsilon>0$. Suppose $E$ is homoclinic with respect to $f$. Then there is a Lipschitz extension $F: I \rightarrow I$ of $f$ with constant $N$ so that $E=\omega_{F}(x)$ is an $\omega$-limit set of $F$ for some $x \in I$.

Proof. From Theorem 2 and Lemma 2 of [BS1], it suffices to show that there is a Lipschitz extension $F: I \rightarrow I$ of $f$ so that for any $x \in E$ and any neighborhood $U$ of $x$, the set $F(U)$ is a neighborhood of $F(x)=f(x)$, with $F \in \operatorname{Lip} N$. To this end, let $\left\{J_{n}\right\}$ be a sequence of open intervals such that diam $J_{n}<1 / n$ and $J_{n} \cap E \neq \emptyset$ for any $n$, and so that every open set intersecting $E$ contains some $J_{n}$. Define by induction continuous extensions $\left\{F_{n}\right\}_{n=0}^{\infty}$ of $f$ to $I$ as follows: Let $F_{0}$ be linear on every interval contiguous to $E$, and put $K_{0}=\emptyset$. If $F_{m}$ and $K_{m}$ are defined, let $K_{m+1}=\left(a_{m+1}, b_{m+1}\right)$ be a complementary interval of $E$ contained in $J_{m}-\left\{K_{0} \cup K_{1} \cup \cdots \cup K_{m}\right\}$. Let $F_{m+1}(x)=F_{m}(x)$ if $x \notin K_{m+1}$, and let $\left|F_{m+1}\left(K_{m+1}\right)\right|=(N-\epsilon / 2)\left(b_{m+1}-\right.$ $\left.a_{m+1}\right)$, with $F_{m+1}\left(K_{m+1}\right)$ centered around $\frac{F_{0}\left(b_{m+1}\right)+F_{0}\left(a_{m+1}\right)}{2}$. Since $f \in$ $\operatorname{Lip}(N-\epsilon)$, we can take $F_{m} \in \operatorname{Lip} N$ for every $m$. Since $\left\|F_{m+1}-F_{m}\right\|<\frac{N}{m}$, we have that $\lim _{n \rightarrow \infty} F_{m}=F$ uniformly, so that $F \in \operatorname{Lip} N$ as well as having the properties we desire.

With Theorems 3.7 and 4.1 in mind, we can now state a corollary that gives us both necessary and sufficient conditions for a set $E$ in $I$ to be a homoclinic attractor for a Lipschitz function.

Corollary 4.2 Let $E$ be a nowhere dense compact set in $I$. Then $E$ is a homoclinic attractor of order $k$ for a Lipschitz function $f: I \rightarrow I$ if and only if

(1) there exists a set of limit points $A=\left\{a_{1}, a_{1}, \ldots, a_{k-1}\right\}$ of $E$, and for each $i=0,1, \ldots, k-1$ a sequence $\left\{E_{n}^{i}\right\}_{n=0}^{\infty}$ of non-empty pairwise disjoint compact subsets of $E$ so that $E \backslash \bigcup_{i, n} E_{n}^{i}=A$, and $\lim _{n \rightarrow \infty} E_{n}^{i}=a_{i}$ for each $i$;

(2) $\frac{a_{i-1}-a_{i}}{M_{1}} \leq a_{i}-a_{i-1}$ for $i=1, \ldots k-2, \frac{a_{k-1}-a_{0}}{M_{1}} \leq a_{0}-a_{1}$, and $\frac{a_{k-2}-a_{k-1}}{M_{1}} \leq a_{k-1}-a_{0}$ for some $M_{1} \in \mathbf{R} ;$

(3) $E_{n}^{i} \stackrel{M_{2}}{\rightarrow} E_{n}^{i-1}$ for $i>0$ and any $n$, and $E_{n}^{0} \stackrel{M_{2}}{\rightarrow} E_{n-1}^{k-1}$ for $n>0$ for some $M_{2} \in \mathbf{R}$; and 
(4) the function $g: E \rightarrow E$ given by $g\left(a_{i}\right)=a_{i-1}$ for $i>0, g\left(a_{0}\right)=a_{k-1}$, $g\left(E_{n}^{i}\right)=E_{n}^{i-1}$ for $i>0$ and any $n, g\left(E_{n}^{0}\right)=E_{n-1}^{k-1}$ for $n>0$, and $g\left(E_{0}^{0}\right)=a_{k-1}$ is Lipschitz, necessarily of constant $M \geq \max \left\{M_{1}, M_{2}\right\}$.

The second and third conditions of our corollary are clearly superfluous in that condition (4) implies them both. The idea to note, however, is that conditions (2) and (3), while necessary, are not themselves sufficient. For $E$ to be a homoclinic attractor of a Lipschitz function, three things must happen. First, we must be able to decompose $E$ into sets $A$ and $E_{n}^{i}$ that develop a homoclinic trajectory. Second, we must be able to accommodate that homoclinic trajectory in the piecewise fashion of conditions (2) and (3) using Theorem 3.7. Finally, the function $g: E \rightarrow E$ implied by (2) and (3) must itself be Lipschitz, that is, the pieces must fit together in such a way that our Lipschitz condition is preserved. Theorem 4.1 then insures the existence of an appropriate extension of $g: E \rightarrow E$ to all of our interval $I$.

Prior to discussing attractors for Lipschitz functions with zero topological entropy, we should recall the following result due to Bruckner and Smítal.

Theorem 4.3 ([BS2]) The set $W$ is an $\omega$-limit set for some continuous function $f$ with zero topological entropy if and only if $W$ is either finite with cardinality a power of two, or of the form $W=Q \dot{\cup} C$ where $Q$ is a Cantor set, $C$ is countable and, if $C \neq \emptyset$, then

(1) $W=\bar{C}$, the closure of $C$, and

(2) card $(C \cap J) \leq 2$ for every interval $J$ complementary to $Q$, and card $(C \cap$ $J) \leq 1$ if $J \cap \overline{\operatorname{conv}} Q=\emptyset$.

Since Lipschitz functions are continuous, we know that those sets which are attractors for Lipschitz functions with zero topological entropy must have the form described in Theorem[BS2, 4.3]. Our final theorem tells us that, so long as we can find a sequence of closed intervals $\left\{T_{k}\right\}$ and Lip $M$ functions $\left\{f_{k}\right\}$ that behave in a fashion similar to what was found in Smítal's Theorem, then our nowhere dense perfect set is an attractor for some $f \in \operatorname{Lip} M$ with zero topological entropy.

Theorem 4.4 Suppose $W$ is a nowhere dense perfect subset of the unit interval. Then $W$ is an $\omega$-limit set for a Lipschitz function $f$ of constant $M$ and zero topological entropy if there exists a sequence of closed intervals $\left\{T_{k}\right\}$ and a sequence of $\operatorname{Lip} M$ functions $\left\{f_{k}\right\}$ defined on $[0,1]$ so that

(1) for each $k,\left\{f_{k}^{i}\left(T_{k}\right)\right\}_{i=1}^{2^{k}}$ are pairwise disjoint and $T_{k}=f_{k}^{2^{k}}\left(T_{k}\right)$;

(2) for each $k, T_{k+1} \cup f_{k+1}^{2^{k}}\left(T_{k+1}\right) \subseteq T_{k}$; 
(3) for each $k, W \subseteq \bigcup_{i=1}^{2^{k}} f_{k}^{i}\left(T_{k}\right)$;

(4) for each $i$ and $k, W \cap f_{k}^{i}\left(T_{k}\right) \neq \emptyset$;

(5) for each $i,\left|f_{k}^{i}\left(T_{k}\right)\right| \rightarrow 0$ as $k \rightarrow \infty$.

Proof. We begin by noting that since $f_{k}$ is already a Lipschitz function with constant $M$ for any $k$, our sequence of functions $\left\{f_{k}\right\}$ is both uniformly bounded and equicontinuous on $[0,1]$. From the Ascoli-Arzelà Theorem, then, there exists a uniformly convergent subsequence $\left\{f_{n_{k}}\right\} \subseteq\left\{f_{n}\right\}$; say, $\lim _{n_{k} \rightarrow \infty} f_{n_{k}}=f$. Then $f$ also satisfies conditions (1) through (5), and $f(W)=W$. Since the set $\{g \in C(I, I): g$ has zero topological entropy $\}$ is closed in $C(I, I), f$ also has zero topological entropy. Now, let $\epsilon>0$. From condition (5) there exists an $N \in \mathbb{N}$ so that $n>N$ implies $\left|f^{i}\left(T_{n}\right)\right|<\epsilon$ for each $i$. Moreover, since $T_{n}=f^{2^{n}}\left(T_{n}\right)$, there exists $x_{n} \in T_{n}$ that is periodic with period $2^{n}$. It follows, then, that $d\left(\omega_{f}\left(x_{n}\right), W\right)<\epsilon$, where $d$ is the Hausdorff metric and $\omega_{f}\left(x_{n}\right)=\left\{x_{n}, f\left(x_{n}\right), \ldots, f^{2^{n}-1}\left(x_{n}\right)\right\}$ is the $\omega$-limit set of $f$ generated by $x_{n}$. We know from [BBHS], however, that the family of $\omega$-limit sets of $f$ is closed with respect to the Hausdorff metric. Since $\omega_{f}\left(x_{n}\right) \rightarrow W$ as $n \rightarrow \infty$, our conclusion follows.

It is worth noting that the requirements of Theorem 4.2 impose a strong measure condition on the set $W$ :

Suppose $W$ satisfies the hypotheses of Theorem 4.2 , and $\mu_{\alpha}(W)$ is either zero, finite, or infinite for $\alpha \in \Phi$. Then together with $W, \mu_{\alpha}(P)$ is either zero, finite, or infinite for any portion $P$ of $W$.

Proof. Suppose $P$ is a portion of $W$. Then there exists $k$ in $\mathbb{N}$ and $j \in$ $\left\{1,2, \ldots 2^{k-1}\right\}$ so that $f^{j}\left(T_{k} \cap W\right) \subseteq P$, and $C \subseteq\left\{1,2, \ldots 2^{k-1}\right\}$ so that $\cup_{j \in C} f^{j}\left(T_{k} \cap W\right) \supseteq P$. Since $f^{j}\left(T_{k}\right)$ is mapped onto $f^{i}\left(T_{k}\right)$ by a Lipschitz map for any $i \in C$, it follows that $\mu_{\alpha}(P)$ and $\mu_{\alpha}\left(T_{k} \cap W\right)$ are together either zero, finite, or infinite for any $\alpha \in \Phi$. But $\cup_{0 \leq i \leq 2^{k-1}} f^{i}\left(T_{k}\right) \supseteq W$, so by the same argument we have that $\mu_{\alpha}(P)$ and $\mu_{\alpha}(W)$ are together either zero, finite, or infinite.

This is in marked contrast to the continuous case, since for any two Cantor sets $E$ and $F$ there exists an order preserving homeomorphism $f: I \rightarrow I$ so that $f(E)=F$. 


\section{References}

[BBHS] A. Blokh, A. M. Bruckner, P. D. Humke and J. Smítal, The space of $\omega$-limit sets of a continuous map of the interval, MSRI Preprint, 063-94, 1994, 1-19.

[BC] A. M. Bruckner and J. G. Ceder, Chaos in terms of the map $x \mapsto$ $\omega(x, f)$, Pac. J. Math., 156 (1992), 63-96.

[BS1] A. M. Bruckner and J. Smítal, The structure of $\omega$-limit sets for continuous maps of the interval, Math. Bohemica, 117 (1992), 42-47.

[BS2] A. M. Bruckner and J. Smítal, A characterization of $\omega$-limit sets of maps of the interval with zero topological entropy, Ergod. Th. and Dynam. Sys., 13 (1993), 7-19.

[BSt] A. M. Bruckner and T. H. Steele, The Lipschitz structure of continuous self-maps of generic compact sets, J. Math. Anal. Appl., 188 (1994), 798-808.

[S] J. Smítal, Chaotic functions with zero topological entropy, Trans. Amer. Math. Soc., 297 (1986), 269-282.

[St] T. H. Steele, The topological structure of attractors for differentiable functions, Real Anal. Ex., 21 (1995), 181-193. 